\title{
FMCW Radar for Small Displacement Detection of Vital Signal Using Projection Matrix Method
}

\author{
Dan Zhang, Masahiko Kurata, and Takayuki Inaba \\ The Graduate School of Informatics and Engineering, University of Electro-Communications, 1-5-1 Chofugaoka, \\ Chofu, Tokyo 182-8585, Japan \\ Correspondence should be addressed to Dan Zhang; zhangdan1227@hotmail.com
}

Received 12 June 2013; Revised 7 October 2013; Accepted 13 November 2013

Academic Editor: Atsushi Mase

Copyright (c) 2013 Dan Zhang et al. This is an open access article distributed under the Creative Commons Attribution License, which permits unrestricted use, distribution, and reproduction in any medium, provided the original work is properly cited.

Small displacement detection has been studied for extracting heart rate signal from the respiratory variation component of the human body with the FMCW radar method. And a new signal processing method of vital signal has been proposed for suppression of unrequired variation components called projection matrix method. We have presented experimental results of small displacement detection to confirm the validity of the method.

\section{Introduction}

Small displacement detection technology in millimetre order at microwave band is expected to a number of applications. Some examples have been proposed such as determining biological signals of heart rate and respiration by noncontact measuring the displacement of the surface of the body and searching survivors under rubble after earthquake by detecting body movements [1-3]. We know that the CW (continuous wave) radar system and UWB pulse radar system have been used for measuring the small displacement [4]. CW radar has the advantages of low power consumption and simple radio architecture. Moreover, CW radar can also cancel out clutter noise by proper adjustment of the radio front-end architecture. The main advantages of the FMCW (frequency modulated continuous wave) radar are simple solid-state transmitters, resistance to interception, and good range resolution. FMCW method performs the velocity and range measurement, and it is considered a good solution for noncontact vital signs detection [1-3].

However, the respiration signal dominates the spectra, and its harmonics may overwhelm the heartbeat signal, making the latter invisible in the spectral analysis sign. The conventional Fourier transform method cannot reliably separate the components. Some signal processing methods are needed to solve this problem. A parametric and cyclic optimization approach, called the RELAX algorithm, has been discussed [5]. The method may take longer computation time to accurately estimate closer signals [6]. Considering these problems, in this paper, we propose a method for suppressing unnecessary periodic fluctuation component with a projection matrix. First we briefly introduce formula of FMCW method and then consider the removal method of the reflected waves from stationary objects that exist in the periphery of the body. Last we have shown the results of the experiment with radar devices that comply with specified low power radio station $24 \mathrm{GHz}$. We have verified the effectiveness of the proposed method for suppressing unnecessary fluctuation component.

\section{Materials and Methods}

2.1. Basic Formula. As we know, the FMCW method uses transmission waves swept linearly with times. From the FMCW principle, the received waves are mixed with the transmission and then remove the sum signal by LPF (low pass filter $5 \mathrm{kHz}$ ); the beat signal can be obtained as follows [7]:

$$
B(t) \cong A \exp \left\{j\left[2 \pi\left(-\frac{2 B R}{c T}-\frac{2 v}{\lambda}\right) t-\frac{4 \pi R}{\lambda}\right]\right\}
$$




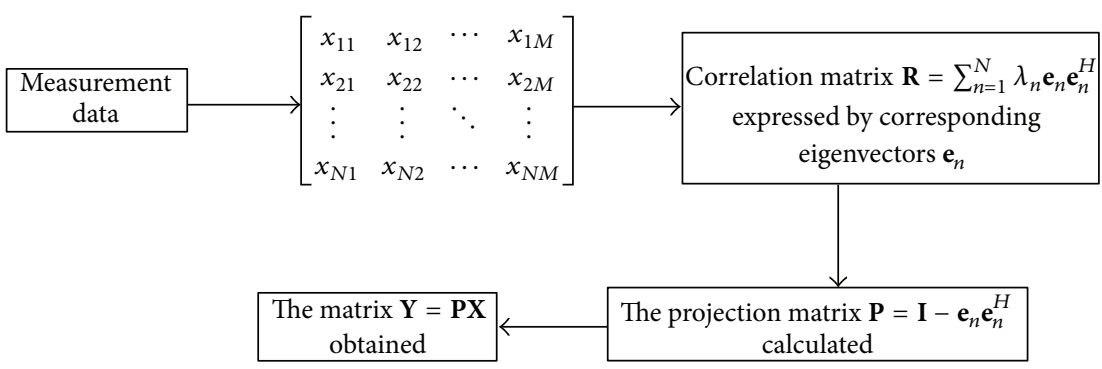

FIgURE 1: Schematic diagram of the projection matrix method.

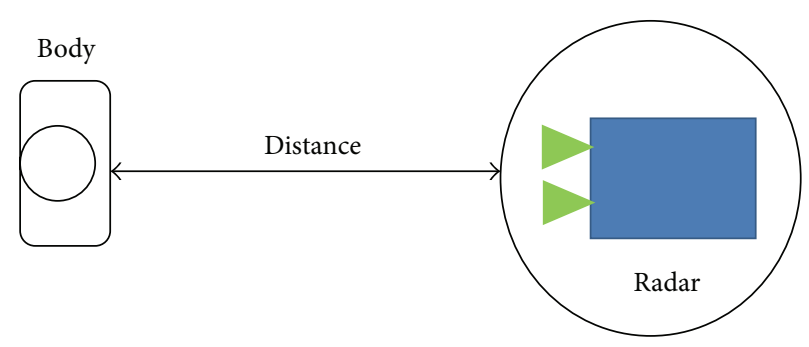

FIGURE 2: Schematic diagram of the experiment.

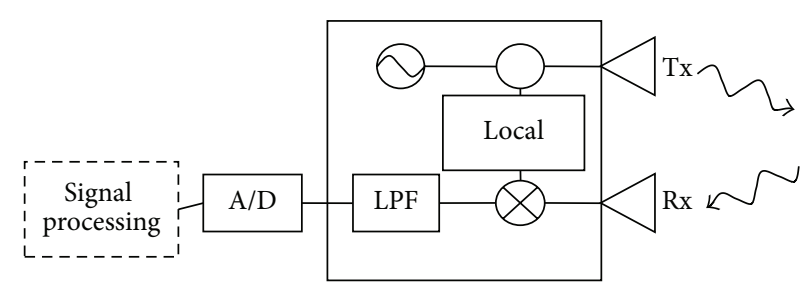

FIGURE 3: Block diagram of the radar system.

Repeating to send signal and obtain a beat signal of (1), we should pay attention to the phase term $4 \pi \cdot R / \lambda$, the phase of the distance $R$ will vary $2 \pi$, according as the distance $R$ varies $\lambda / 2$. Thus, it is possible to measure small change in the distance $R$ by the phase term with high sensitivity. The phase values will be calculated by $I$ and $Q$ signals of the beat signal and then obtained by Fourier transform.

2.2. Interference Removal. If there are some other stationary objects around the body, they may interfere with the reflected wave received. Considering the interference, (1) can be converted to

$$
\begin{aligned}
B(t) \cong & A \exp \left\{j\left[2 \pi\left(-\frac{2 B R}{c T}-\frac{2 v}{\lambda}\right) t-\frac{4 \pi R}{\lambda}\right]\right\} \\
& +A_{s} \exp \left\{j\left[2 \pi\left(-\frac{2 B R_{s}}{c T}\right) t-\frac{4 \pi R_{s}}{\lambda}\right]\right\} .
\end{aligned}
$$

With the passage of time, the second term in the equation for the objects will have no variation, so we can remove the term by subtraction [7].

2.3. Projection Matrix. The heart rate signal must be extracted from the respiratory variation component, when we measure the displacement of the body surface. The method of HPF (high pass filter) has been used for separating heart signal components from a respiratory fluctuation component $[8,9]$. However, this method cannot separate the harmonic components from the respiratory variation, and it is hard to reproduce heart rate waveform and measure the heart rate [10]. So we propose a method for suppressing unnecessary periodic fluctuation component with projection matrix [11]. The method can reduce the effects of harmonics. The schematic diagram of the method is shown in Figure 1.

First, we obtain the sampling period of unnecessary fluctuation component by performing Fourier transform for the measurement data. We can obtain the vector $\mathbf{X}=$ $\left[x_{1}, x_{2}, \ldots, x_{m}\right]$, where $x$ express sampling dates whose period number is $N$; that is, each $x_{i}$ has $N$ sampling dates.

From the vector $\mathbf{X}$, the correlation matrix can be calculated as follows:

$$
\mathbf{R}=\left\langle\mathbf{X} \mathbf{X}^{H}\right\rangle,
$$

where the operation \langle\rangle represents the average.

The correlation matrix $\mathbf{R}$ can be expressed with eigenvalue decomposition as follows:

$$
\mathbf{R}=\sum_{n=1}^{N} \lambda_{n} \mathbf{e}_{n} \mathbf{e}_{n}^{H},
$$



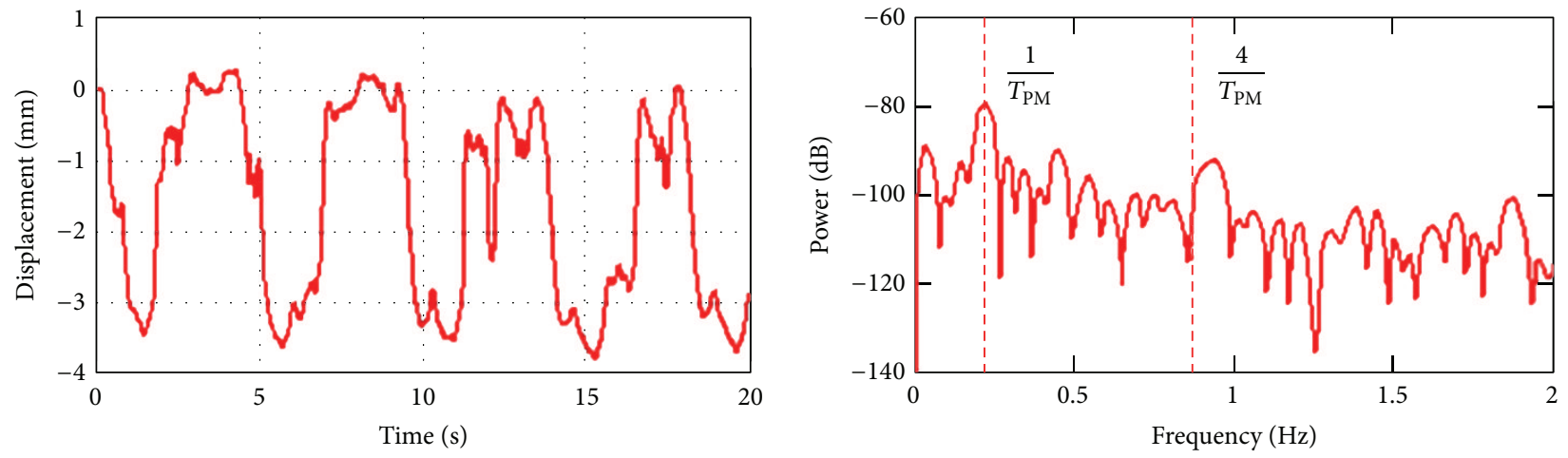

(a) Conventional method
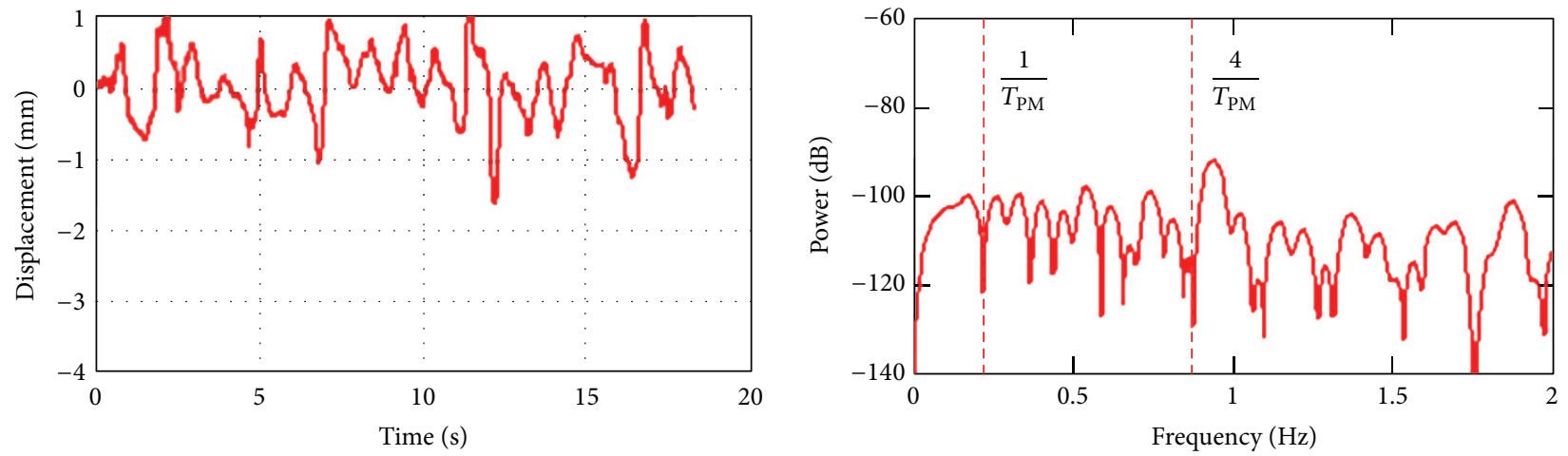

(b) Projection matrix method

FIGURE 4: Signal processing results at the distance of $30 \mathrm{~cm}$.
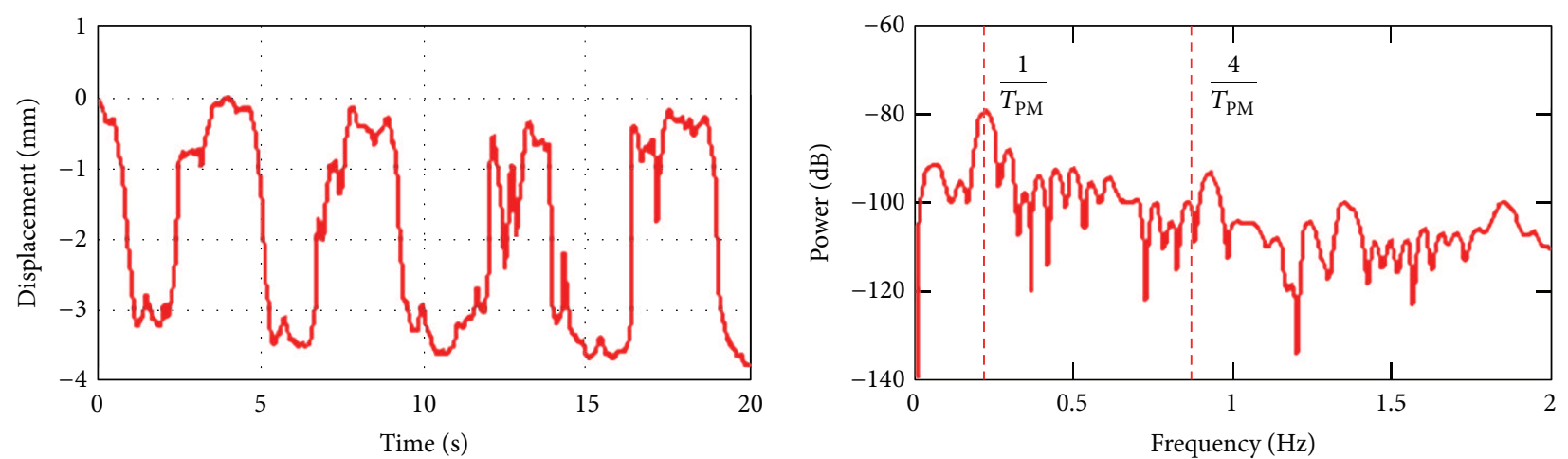

(a) Conventional method
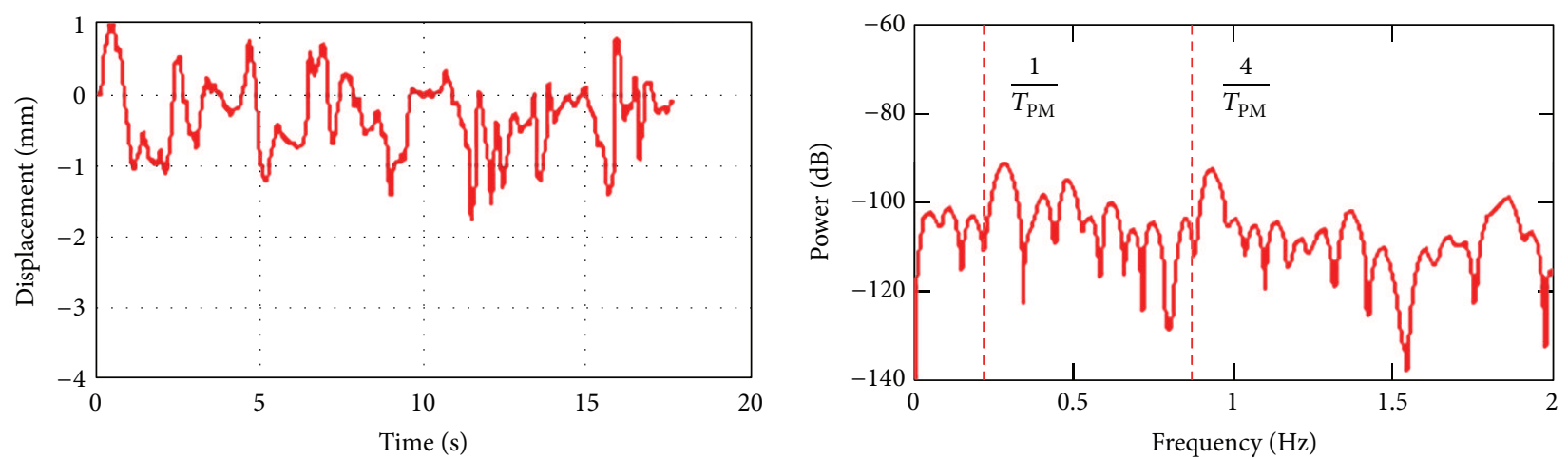

(b) Projection matrix method

FIGURE 5: Signal processing results at the distance of $40 \mathrm{~cm}$. 

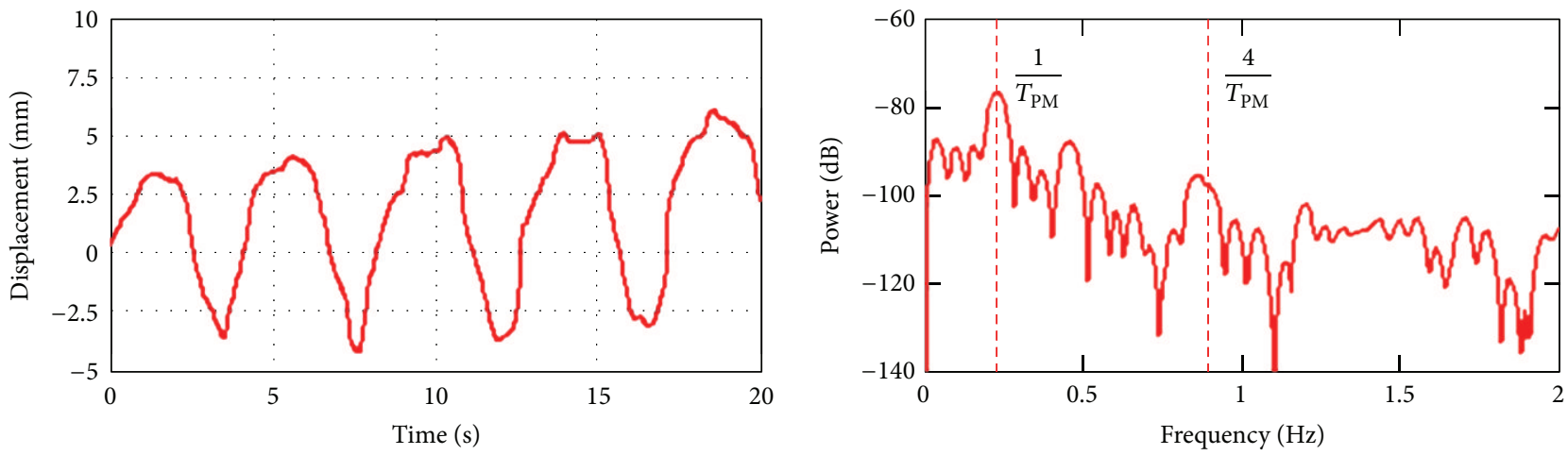

(a) Conventional method
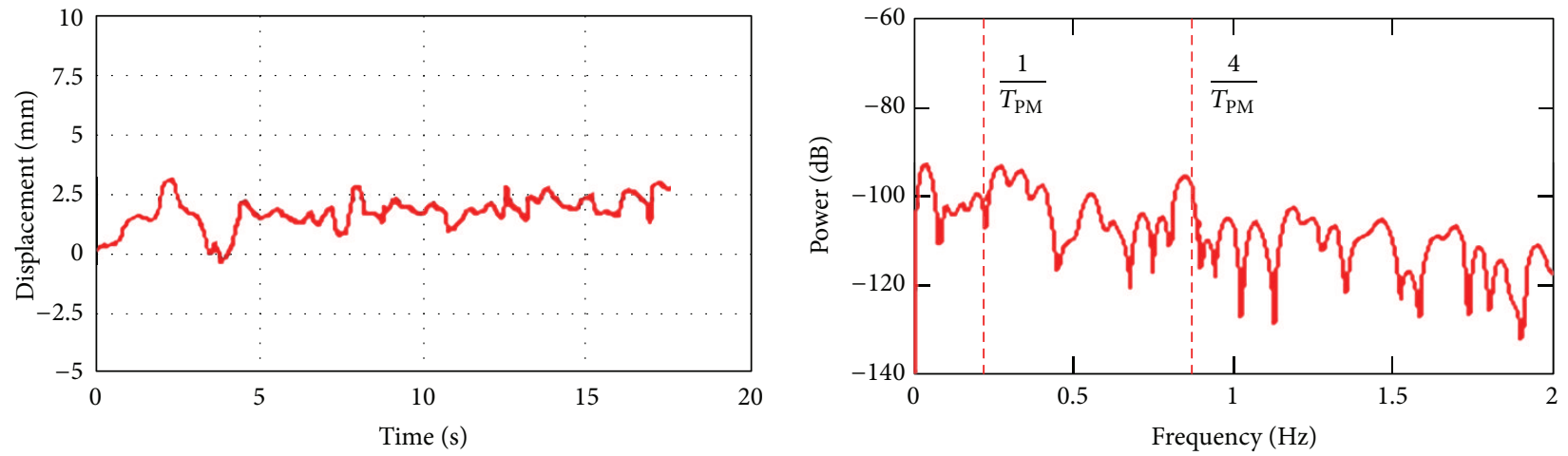

(b) Projection matrix method

FIGURE 6: Signal processing results at the distance of $200 \mathrm{~cm}$.

where $\lambda_{n}$ are the eigenvalues and $\mathbf{e}_{n}$ are the corresponding eigenvectors.

Because the vector $\mathbf{X}$ is divided by the period of unnecessary fluctuation component, the largest eigenvalue corresponds to the fluctuation component. The matrix $\mathbf{S}$ corresponding to the unnecessary fluctuation component is obtained from the eigenvector $\mathbf{e}_{i}$ corresponding to the maximum eigenvalue as follows:

$$
\mathbf{S}=\mathbf{e}_{i} \mathbf{e}_{i}^{H} .
$$

Then, the projection matrix for the suppression of unnecessary fluctuation component is defined as

$$
\mathbf{P}=\mathbf{I}-\mathbf{S}
$$

where $\mathbf{I}$ is a $N \times N$ identity matrix. We perform the following processing for the vector $\mathbf{X}$ to suppress unnecessary variation component:

$$
\mathbf{Y}=\mathbf{P X}
$$

The projection matrix $\mathbf{P}$ is orthogonal to the matrix $\mathbf{S}$ corresponding to unnecessary fluctuation component of the vector $\mathbf{X}$.

\section{Results and Discussion}

The experiment is performed using the software radar developed by our laboratory as shown in the Figure 2. The target is a human body. The propagation frequency is $24.15 \mathrm{GHz}$, sampling frequency is $10 \mathrm{kHz}$, sweep bandwidth is $72 \mathrm{MHz}$, and sweep time is $25.6 \mathrm{~ms}$ in the FMCW method, respectively. The block diagram of the radar system has been shown in Figure 3. Usually, the data may be measured in three conditions such as the orientation of the body, the distance to radar, and the radar irradiation position on body. In this time, the front of body is facing the radar and the irradiation position is selected as the largest displacement caused by heart beating. The distance to radar is changed to $30 \mathrm{~cm}$, $40 \mathrm{~cm}$, and $200 \mathrm{~cm}$ in our measurement, respectively.

The experiment results using the FMCW method are shown in Figures 4(a), 5(a), and 6(a), and after using the projection matrix method, the results are shown in Figures 4(b), 5(b), and 6(b), respectively. First we obtain the time domain as shown in the left figures and then convert them to spectrum as shown in the right figures. We can easily know that the high peak near $0.25 \mathrm{~Hz}$ is the respiration rate in each Figure (a), and we also obviously find that the heartbeat rate is about $0.9 \mathrm{~Hz}$ in each Figure (b). Especially, the signal is relatively weak in the distance of $200 \mathrm{~cm}$, and the heartbeat is not obvious. After importing the method, it is possible to clearly show the heart signal.

Using the proposed projection matrix method, even if the frequency of the heart rate and respiration frequency are close to each other, it is possible to separate and extract the heart rate signal. 


\section{Conclusions}

We have performed small displacement measurement with the FMCW radar method for extracting the heart rate signal of the human body. And we have proposed a signal processing method for suppressing unnecessary periodic fluctuation component with the projection matrix. From the experiment results, the effectiveness of the method has been confirmed.

\section{References}

[1] C. Li, Y. Xiao, and J. Lin, "Experiment and spectral analysis of a low-power $\mathrm{K}$ a-band heartbeat detector measuring from four sides of a human body," IEEE Transactions on Microwave Theory and Techniques, vol. 54, no. 12, pp. 4464-4471, 2006.

[2] M. Wakayama, H. Ezaki, I. Arai, and T. Miwa, "Non-contact measurement of heart rate using FM-CW radar," IEICE Technical Report SANE2005-3, 2005, (Japanese).

[3] I. Arai, "Life-detection radar for rescue purpose," IEICE Technical Report SANE99-100, 2000, (Japanese).

[4] C. Li and J. Lin, "Recent advances in doppler radar sensors for pervasive healthcare monitoring," in Proceedings of the AsiaPacific Microwave Conference (APMC '10), pp. 283-290, WE4C5, Yokohama, Japan, December 2010.

[5] C. Li, J. Ling, J. Li, and J. Lin, "Accurate doppler radar noncontact vital sign detection using the RELAX algorithm," IEEE Transactions on Instrumentation and Measurement, vol. 59, no. 3, pp. 687-695, 2010.

[6] N. Shahid, D. Fang, W. Sheng, and X. Ye, "The high resolution estimate-a comparative study," in Proceedings of the International Conference on Computational Electromagnetics and its Applications (ICCEA '99), pp. 262-265, 1999.

[7] M. Kurata and T. Inaba, "Basic study of small displacement detection using microwave radar," IEICE Technical Report SANE2012-135, 2013.

[8] B. Lohman, O. Boric-Lubecke, V. M. Lubecke, P. W. Ong, and M. M. Sondhi, "A digital signal processor for doppler radar sensing of vital signs," IEEE Engineering in Medicine and Biology Magazine, vol. 21, no. 5, pp. 161-164, 2002.

[9] M. Wakayama, H. Ezaki, I. Arai, and T. Miwa, "Non-contact measurement of heart rate using FM-CW radar," IECE Technical Report SANE2005-3.

[10] G. Lu, F. Yang, X. Jing, and J. Wang, "Contact-free measurement of heartbeat signal via a doppler radar using adaptive filtering," in Proceedings of the 2nd International Conference on Image Analysis and Signal Processing (IASP '10), pp. 89-92, April 2010.

[11] T. J. Nohara, P. Weber, and A. Premji, "Adaptive mainbeam jamming suppression for multi-function radars," in Proceedings of the IEEE National Radar Conference, pp. 207-212, May 1998. 

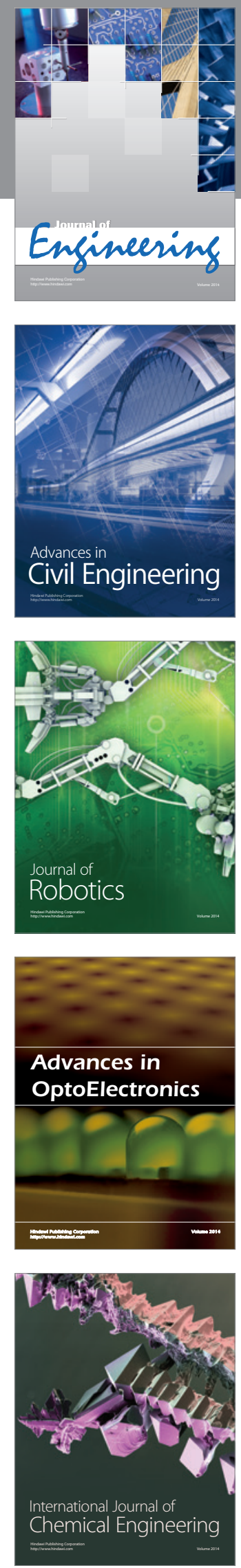

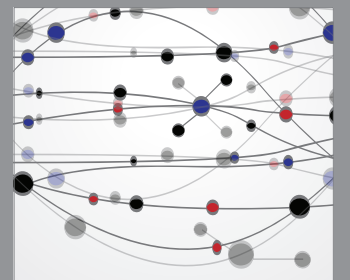

The Scientific World Journal
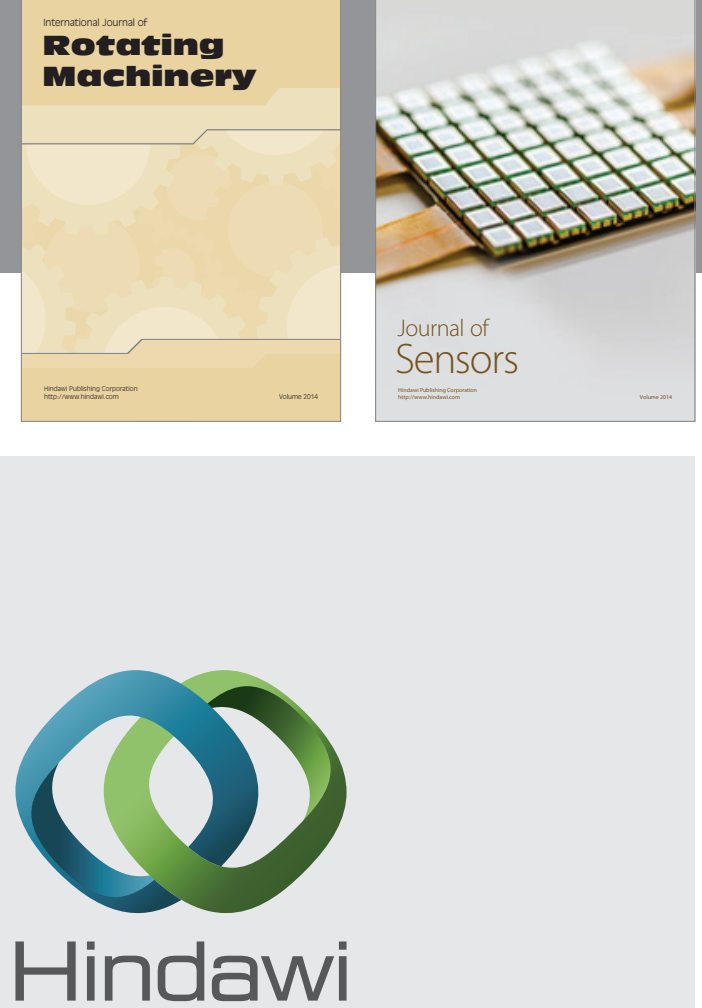

Submit your manuscripts at http://www.hindawi.com
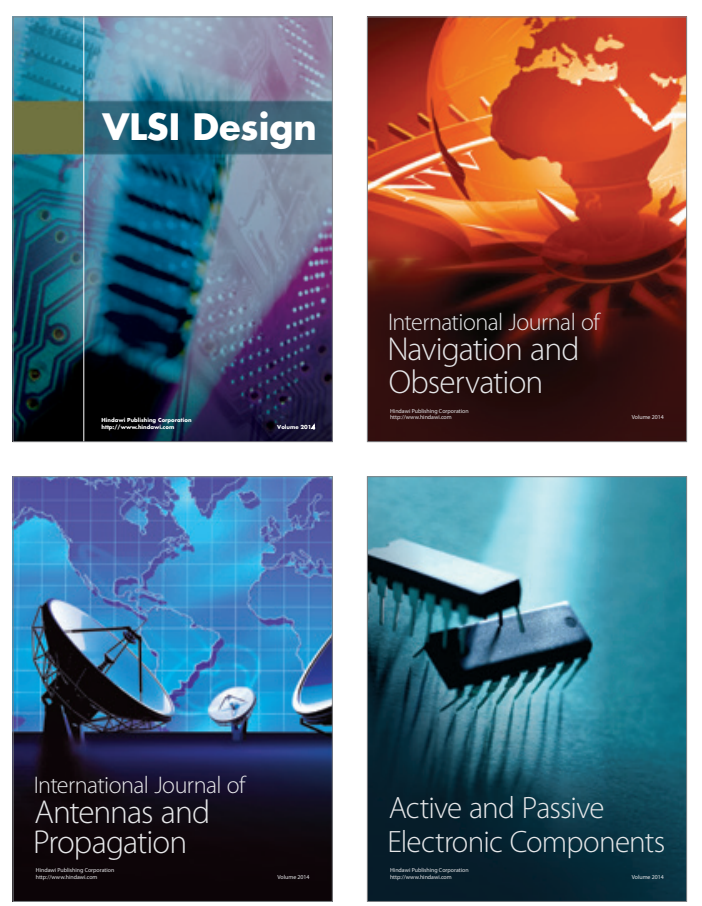
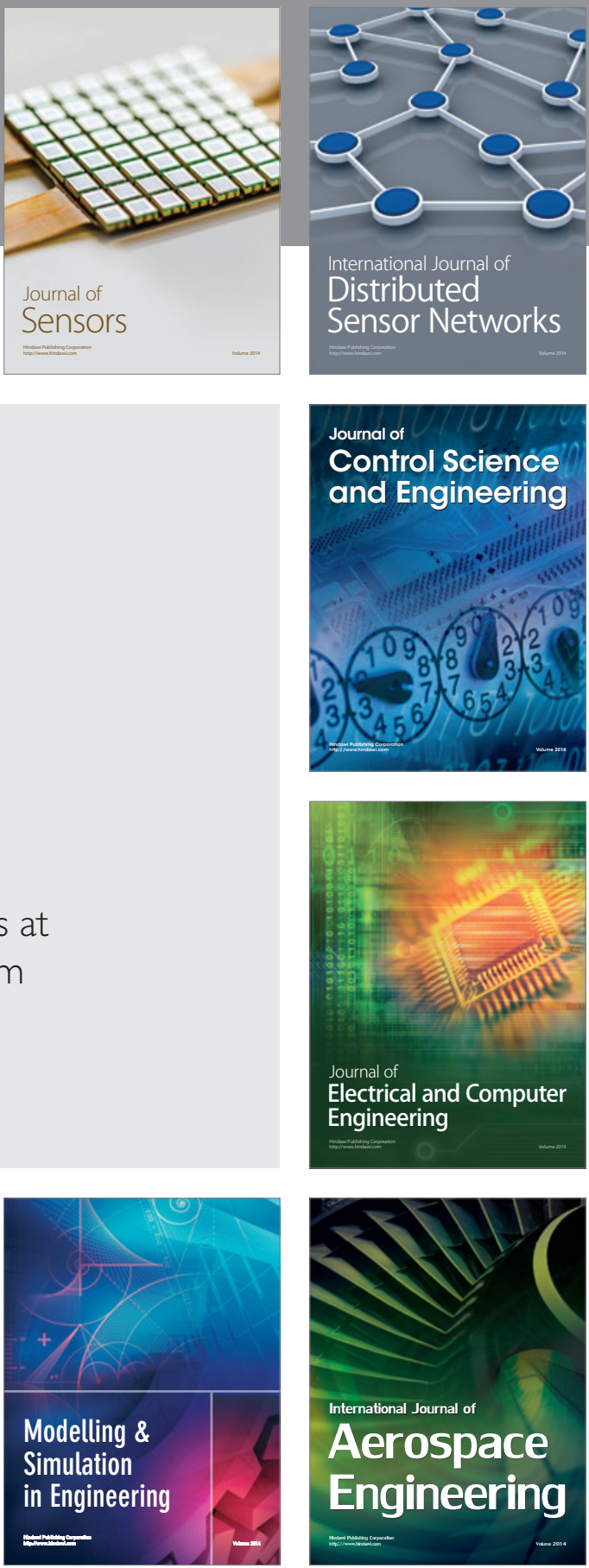

Journal of

Control Science

and Engineering
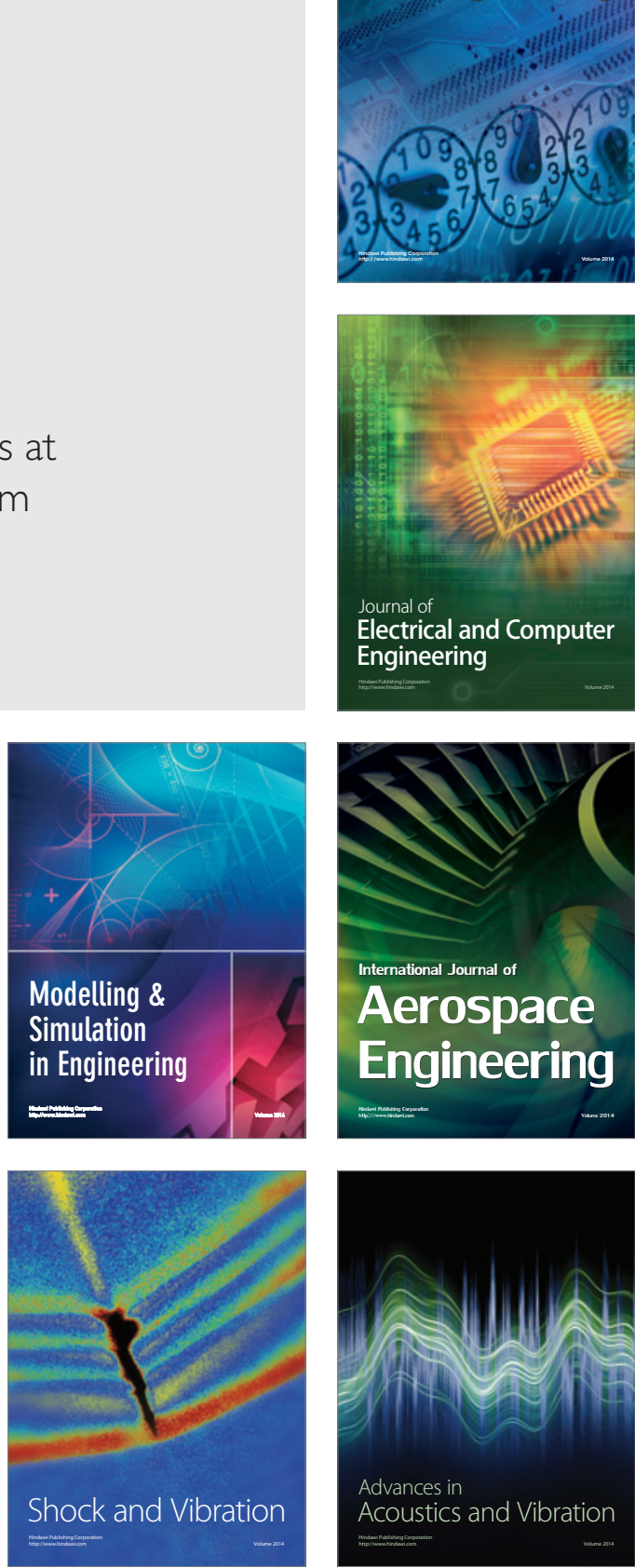